\title{
Cohesive Crack Analysis of Size Effect for Samples with Blunt Notches and Generalized Size Effect Curve for Quasi-Brittle Materials
}

\author{
Giovanni Di Luzio ${ }^{\mathrm{a}}$, Gianluca Cusatis ${ }^{\mathrm{b}}$ \\ ${ }^{a}$ Department of Civil and Environmental Engineering, Politecnico di Milano, Milan, \\ Italy. \\ ${ }^{b}$ Department of Civil and Environmental Engineering, Northwestern University, \\ Evanston (IL), USA.
}

\begin{abstract}
This paper deals with the study of size effect on structural strength for quasibrittle materials under mode I fracture conditions. By using a linear cohesive crack model, accurate numerical simulations were performed to compute the size effect curves for two test configurations - namely, the three-point bending test with span-to-depth ratio equal to 3 and the center crack panel test featuring sharp notches and blunt notches whose width is also scaled with the specimen dimension.

The analysis of the results shows that, as the specimen size tends to infinity, the asymptotic behavior depends on the type of notch. For sharp notches the size effect curve tends asymptotically to Bažant's Size Effect Law. On the contrary, for blunt notches the size effect curve tends to a horizontal asymptote corresponding to the elastic limit. The elastic limit can be calculated by the tensile strength reduced by the stress concentration factor at the tip of the blunt notch and it depends on the geometry of the specimen.
\end{abstract}


Furthermore, the numerical results were utilized to derive the expression of a Generalized Cohesive Size Effect Curve (GCSEC) which agrees well with Bažant's Universal Size Effect Law (USEL) and with some experimental data gathered from the literature.

Keywords: Cohesive Crack Model, Size Effect, Quasi-Brittle Materials, Cohesive Fracture

\section{Introduction}

Quasi-brittle materials (concrete, rock, ceramics, etc.) show dependence of the structural strength, $\sigma_{N}$, on the structural size $D$. This phenomenon is referred to in the literature as size effect. Typically, the structural strength is defined as a normalized measure of the load carrying capacity (peak load), and its definition depends on the structural geometry and loading configurations [4].

Based on the cohesive crack model, the size effect for mode I fracture can be described through the following equation $[4,3,5]$ :

$$
\left(\frac{f_{t}^{\prime}}{\sigma_{N}}\right)^{2}=\Phi\left(D / l_{c h}\right)
$$

where $l_{c h}=E G_{F} / f_{t}^{\prime 2}$ is Hillerborg's characteristic length [2], $f_{t}^{\prime}=$ tensile strength, $E=$ Young's modulus, and $G_{F}=$ fracture energy. The size effect relationship represented symbolically by Eq. 1 is termed Cohesive Size Effect Curve (CSEC) [1].

Various authors developed [1,3] analytical expressions of the function $\Phi$ by fitting the results of numerical simulations of cohesive crack propagation in geometrically similar samples of increasing size. However, these studies analyzed the case of sharp notches only. 
Previous analytical and numerical studies [3], suggested that the CSEC depends on structural shape, load configuration and boundary conditions, as well as the type of the cohesive stress versus crack opening relationship, a.k.a. the Cohesive Crack Law (CCL).

Cusatis and Schauffert [1] analyzed five different structural configurations of sharp-notched specimens (direct tension on center crack panel, direct tension on double and single edge notch panels, and three-point bending with two different span-to-depth ratios). They verified that for small sizes, the CSEC tends to the plastic limit. Furthermore, they discovered that the CSEC curves feature a straight line asymptotes which is independent of structural geometry, boundary conditions, and loading configurations when normalized by the Linear Elastic Fracture Mechanics (LEFM) dimensionless energy release parameters, $g_{0}$ and $g_{0}^{\prime}$ [4], corresponding to each particular case. They showed also that such asymptote coincides with Bažant's Size Effect Law (SEL) [6], which can be expressed as:

$$
\frac{1}{g_{0}^{\prime}}\left(\frac{f_{t}^{\prime}}{\sigma_{N}}\right)^{2}=\frac{g_{0} D}{g_{0}^{\prime} l_{c h}}+\frac{c_{F}}{l_{c h}}
$$

where $c_{F}$ is the so called Effective Fracture Process Zone Length (EFPZL). For $c_{F}=0$, the SEL coincides with the size effect provided by LEFM

$$
\frac{1}{g_{0}^{\prime}}\left(\frac{f_{t}^{\prime}}{\sigma_{N}}\right)^{2}=\frac{g_{0} D}{g_{0}^{\prime} l_{c h}}
$$

It is worth pointing out that Eqs. 2 and 3 only apply to structures with positive geometries that are characterized by a dimensionless energy release rate that increases as the normalized crack length increases: $g_{0}^{\prime}>0$.

Building upon the work in $[5,11]$, Cusatis and Schauffert [1] were able to show that, despite discordant results available in the literature $[8,9,10,5]$, 
$c_{F} / l_{c h}$ is independent of geometrical and loading configurations and it is equal to 0.44 for a linear CCL. This condition, namely that the EFPZL is a material property, had been assumed previously in typical SEL derivations.

Finally, the results in [1] highlighted that, at the peak load, the Fracture Process Zone (FPZ) length, i.e. the length of the ligament portion with cohesive stresses, and notch tip cohesive stress converge for increasing size to the constant values of $0.71 l_{c h}$ (for linear softening) and zero, respectively, regardless of the structural configuration.

The objective of the study discussed in this paper was to build upon previous work and to provide a more general formulation of the cohesive size effect curve to include structural configurations with blunt notches. Such formulation, entitled Generalized Cohesive Size Effect Curve (GCSEC) can be derived by fitting accurate numerical simulations of size effect that are

discussed next. It is worth pointing out that an extensive comparison of the numerical results with experimental results to validate the adoption of the cohesive crack law was outside the scope of this paper as it had been pursued by many other authors before. In this work, the cohesive crack model, particularly in its linear version, was assumed to be a valid model to simulate the fracturing behavior of a certain class of quasi-brittle materials.

\section{Numerical simulations with Cohesive Crack Law}

In this study two different structural configurations were investigated: the direct tension on center crack panel (CCP) and the three-point bending (TPB) with a span/depth ratio $S / D=3$, shown in Fig. 1a and Fig. 1b, respectively. For these configurations, the structural strength can be defined 
as $\sigma_{N}=P_{u} / D t$ for CCP and $\sigma_{N}=1.5(S / D) P_{u} / D t$, for TPB, where $P_{u}=$ peak load, $D=$ panel depth, $t=$ panel thickness, and $S=$ beam span $[4,12]$.
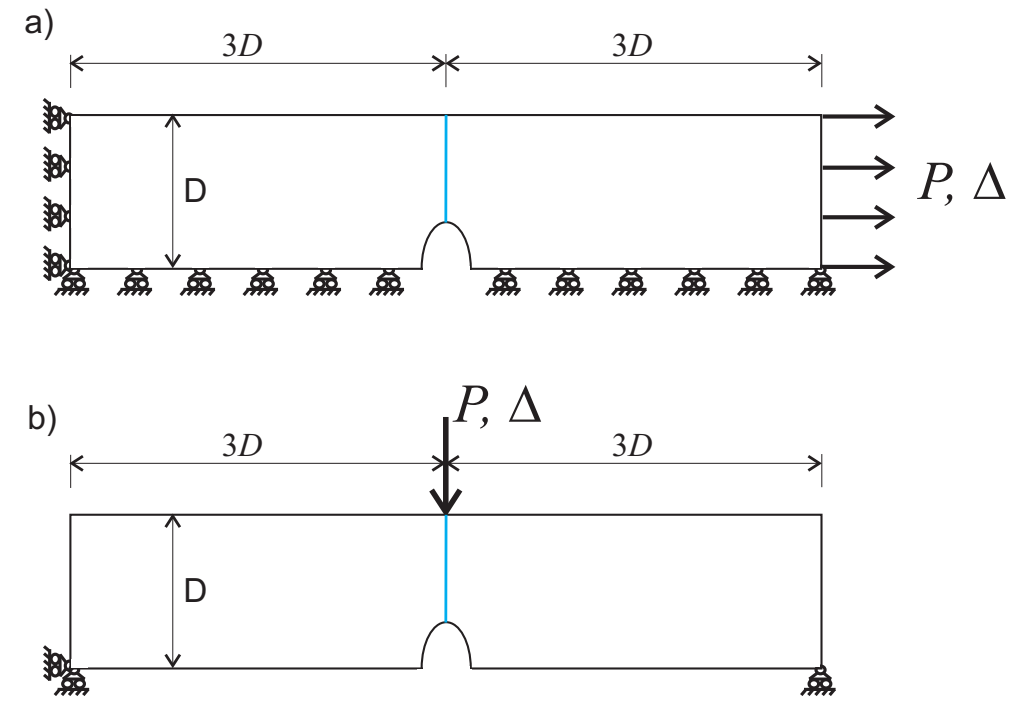

Figure 1: Test configurations and boundary conditions: a) center crack panel (CCP); b) three-point bending (TPB).

The numerical simulations were performed by using standard Finite Element Method (FEM) techniques. The bulk of the discretization (Fig. 2b) was modeled with eight-node, elastic, iso-parametric elements [13], and the crack line was modeled with cohesive interface elements [14] governed by a linear CCL.

The FEM relies on discretizing the continuum domain into finite elements and its accuracy depends greatly on the number of elements used in the discretization. As the mesh is progressively refined, the solution improves and converges to the exact one. However, there are situations where the solution converges poorly with mesh refinement. This is the case when a stress 
singularity exists, for example, at the tip of a sharp notch. As the mesh is refined, the stress at the singular point keeps increasing but it is always a poor approximation of the singularity. Special FE element formulations can be used to capture the stress singularity, which, however, remains problematic in the case of cohesive stress analysis in which the tensile strength, governing crack initiation, has a finite value. These special formulations consist of finite elements allowing strong discontinuities in the displacement field and two broad families can be identified in terms of the support of the enriching discontinuous displacement modes: (1) the Embedded enrichment Finite Element Method (E-FEM) in which the support for each mode is in the element formulation $[15,16,17]$; (2) the eXtended Finite Element Method (X-FEM) in which a nodal enrichment is introduced and the support of each mode is the one of a given nodal shape function $[18,19,20]$.

A different situation arises when the notch is not perfectly sharp and features a small curvature radius. In this case, the stress is not infinite anymore, the stress singularity disappears and a finite stress concentration takes place.

A stress concentration gives similar numerical challenges as stress singularities but the convergence is guaranteed provided that the mesh is sufficiently refined. The level of refinement depends on the radius of curvature of the notch: the smaller it is, the more refined the mesh must be.

Theoretically, to overcome the problems inherent in cohesive crack simulations with sharp notches one could simulate the peak load behavior as the limit of elliptical notches as the elliptical aspect ratio $a / b$ (ratio of the longer-to-shorter dimensions), tends to infinity (Fig. 2a). While theoretically 
correct, this approach incurs also in numerical difficulties for high values $a / b$. Numerically, the best numerical response in presence of sharp notches can be obtained by modeling notches of small finite width, $b$, and a semi-circular tip (Fig. 2a, bottom right). The notch width, $b$, has to be kept constant and not to be scaled with specimen size. Although this violates the geometrical similarity of the specimens, it was shown [1] to provide an excellent approximation of sharp notch cohesive behavior if $b / l_{c h} \ll 1$.

To study the effect of blunt notches, numerical simulations were performed with geometrical similar specimens featuring semi-elliptic notches. The notch geometry was characterized by an semi-elliptical curve with the two semi-axis representing the half-notch width, $b$, and the notch depth, $a$, as shown in Fig. 2a. The perfect geometrical similarity was obtained by by scaling both notch width, $b$, and notch depth, $a$, with the specimen size. The calculations were carried out for six aspect ratios of the notch: namely, $a / b=0.5,1.0,2.0,5.0,8.0$, and 15 (Fig. 2a).

For the reasons discussed above the resolution of finite element meshes utilized to simulate the crack propagation with the cohesive crack model must be very fine especially in the region close to the notch tip as shown in Fig. 2b. In all the simulations, the size of the interface elements ahead of the notch tip and for the entire length of the Fracture Process Zone (FPZ), was not scaled with the specimen size and it was kept within the relatively small range of 0.5-1.0 mm (Fig. 2b). In general, for the smaller specimen sizes the FPZ was modeled with no less than 50 interface elements. For the larger sizes, instead, the FPZ was modeled with up to five hundred interface elements. This ensured a similar resolution of the FPZ (which does not scale 
a)

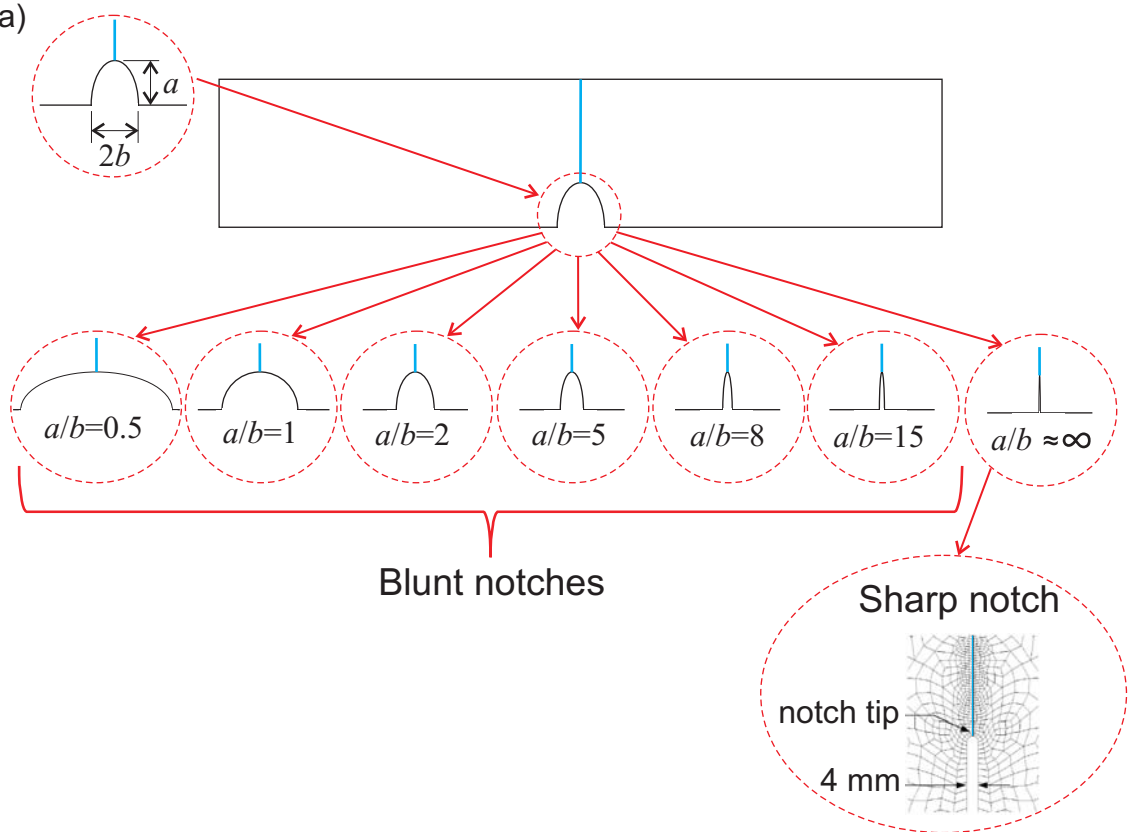

b)
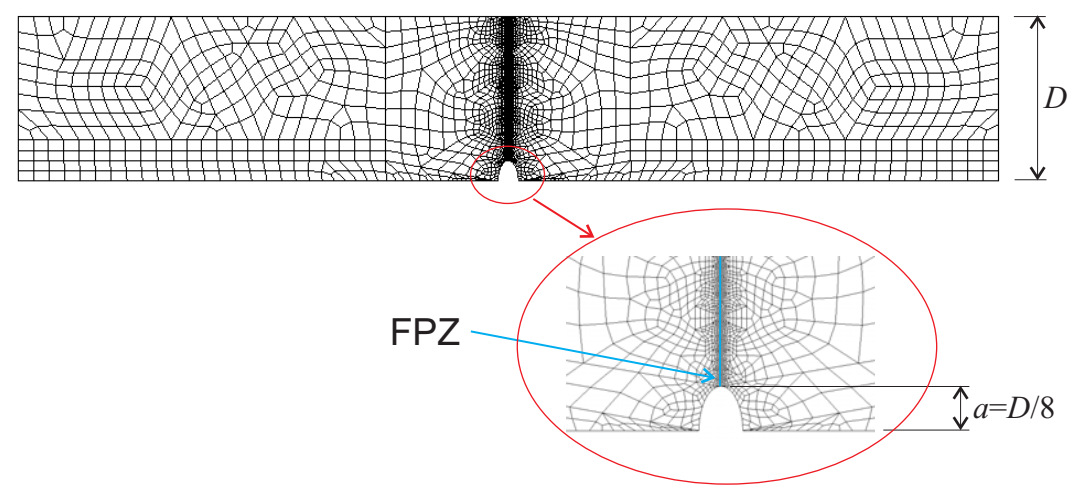

Figure 2: (a) Typical semi-elliptical notch geometries used in the simulations. (b) Example of the FE mesh of the simulated specimens including zoom-in of the notch tip to show the mesh refinement required to capture correctly the stress concentration.

with specimen size) across all specimen sizes.

For each aspect ratio, the peak load was determined for sixteen sizes from 
$D=5 \mathrm{~mm}$, doubling 15 times up to $D=163,840 \mathrm{~mm}$. In all cases, the ratio between the notch depth $a$, and the panel depth $D$, was held constant at $\alpha=a / D=0.125$. The numerical analyses were performed with a linear CCL and the following material properties: $E=24,000 \mathrm{~N} / \mathrm{mm}^{2}, G_{F}=0.05$ $\mathrm{N} / \mathrm{mm}, f_{t}^{\prime}=2.8 \mathrm{~N} / \mathrm{mm}^{2}$, corresponding to $l_{c h}=153 \mathrm{~mm}$. It is worth observing that the size effect curve does not depend on the specific material properties used in the calculations.

The calculated size effect curves are shown in a log-log plot in Fig. 3a and Fig. 4a for the CCP and TPB configurations, respectively. In addition to the size effect curves, the plastic limit, the LEFM limit, and the elastic limits for each notch aspect ratio, are shown in the plots. The elastic limit is defined as a stress state where the normal stress at the tip of the notch first reaches the tensile strength, $f_{t}^{\prime}$, and it corresponds to the cohesive crack initiation. The nominal stress associated with the elastic limit can be calculated using the stress concentration factors at the notch tip: $\sigma_{x x}^{t i p}=k \sigma_{N}$. Since at the elastic limit $\sigma_{x x}^{t i p}=f_{t}^{\prime}$, the elastic nominal stress is $\sigma_{N e}=f_{t}^{\prime} / k$ and its normalized value is $\sigma_{N e} / f_{t}^{\prime}=1 / k$.

For the tip of an elliptical hole in a CCP geometry (Fig. 1a), the stress concentration factor $k$ in a finite-width plate and for $0.5 \leq a / b \leq 10.0$ can be calculated approximately with the following equations [24]: $k=\left(C_{1}+\right.$ $\left.C_{2} \alpha+C_{3} \alpha^{2}+C_{4} \alpha^{3}\right) /(1-\alpha), C_{1}=1.0+2.0(a / b), C_{2}=-0.351+0.21 \sqrt{a / b}-$ $2.483(a / b), C_{3}=+3.621-5.183 \sqrt{a / b}+4.494(a / b)$, and $C_{4}=-2.27+$ $5.204 \sqrt{a / b}-4.011(a / b)$.

For the $a / b$ equal to $0.5,1.0,2.0,5.0,8.0$, and $15 k$ equals $2.094,3.071$, $5.038,10.976,16.933$ and 30.862 , respectively, and the corresponding normal- 
ized elastic limit, $1 / k$, is $0.4775,0.3257,0.1985,0.0911,0.0591$ and 0.0324 . For the unnotched case, $a / b=0, k=1$. It is worth reminding that in the CCP geometry with infinite plate the stress concentration factor $k$ is $k=1.0+2.0(a / b)$.

These analytical values were found to match very well the ones computed numerically.

for the TPB configuration the normalized nominal stress at the elastic limit were obtained numerically as $0.5568,0.3736,0.0964$, and 0.0625 for notch aspect ratios of $0.5,1.0,5.0$, and 8.0, respectively. For the aspect ratio equals to 0 the nominal strength coincides with the modulus of rupture, which for pure bending and with the adopted definition of the nominal stress corresponds to the tensile strength. However, for TPB the modulus of rupture is expected be to be larger because the stress distribution along the central cross section is different from that in pure bending [23]. According to Timoshenko and Goodier [22] the maximum tensile stress is $\sigma_{\text {tip }}^{\text {ela }}=\beta \sigma_{N}$ with $\beta=(1-0.1773 D / S)^{-1}$. For the geometry considered in this study one has $\beta=[1-0.1773(1-\alpha) / 3]^{-1}=1.054$. At the elastic limit, $\sigma_{\text {tip }}^{\text {ela }}=f_{t}^{\prime}$, one obtains $\sigma_{N e}=\beta(1-\alpha)^{2} f_{t}^{\prime}=0.807 f_{t}^{\prime}$.

As expected, the elastic limits are size-independent (they only depend on $\alpha$ and $a / b$ ) and they are plotted as horizontal straight lines in Fig. 3a and 4a, for the TPB and CCP configurations, respectively.

The same plots show the plastic limits as horizontal lines. Furthermore, it is worth noting that, as shown below, the plastic limit is independent on the notch aspect ratio and, consequently, one single horizontal straight line is common to all notch aspect ratios. 

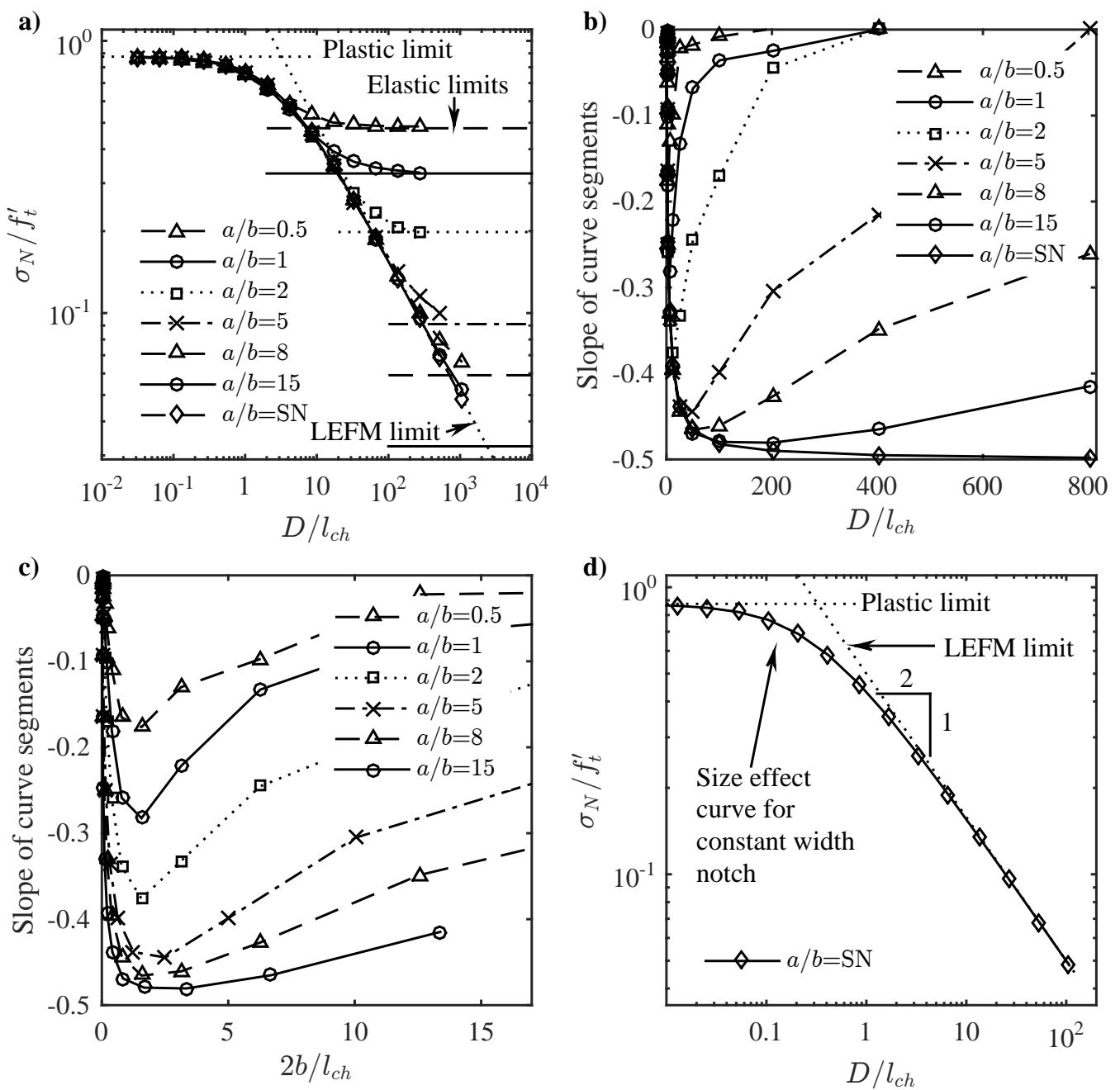

Figure 3: CCP Configuration: (a) size effect curves for elliptically notched geometries, (b) slope of size effect curves versus normalized size, (c) slope of size effect curves versus normalized width of elliptical notch, and (d) size effect curve for constant-width notch geometry. 

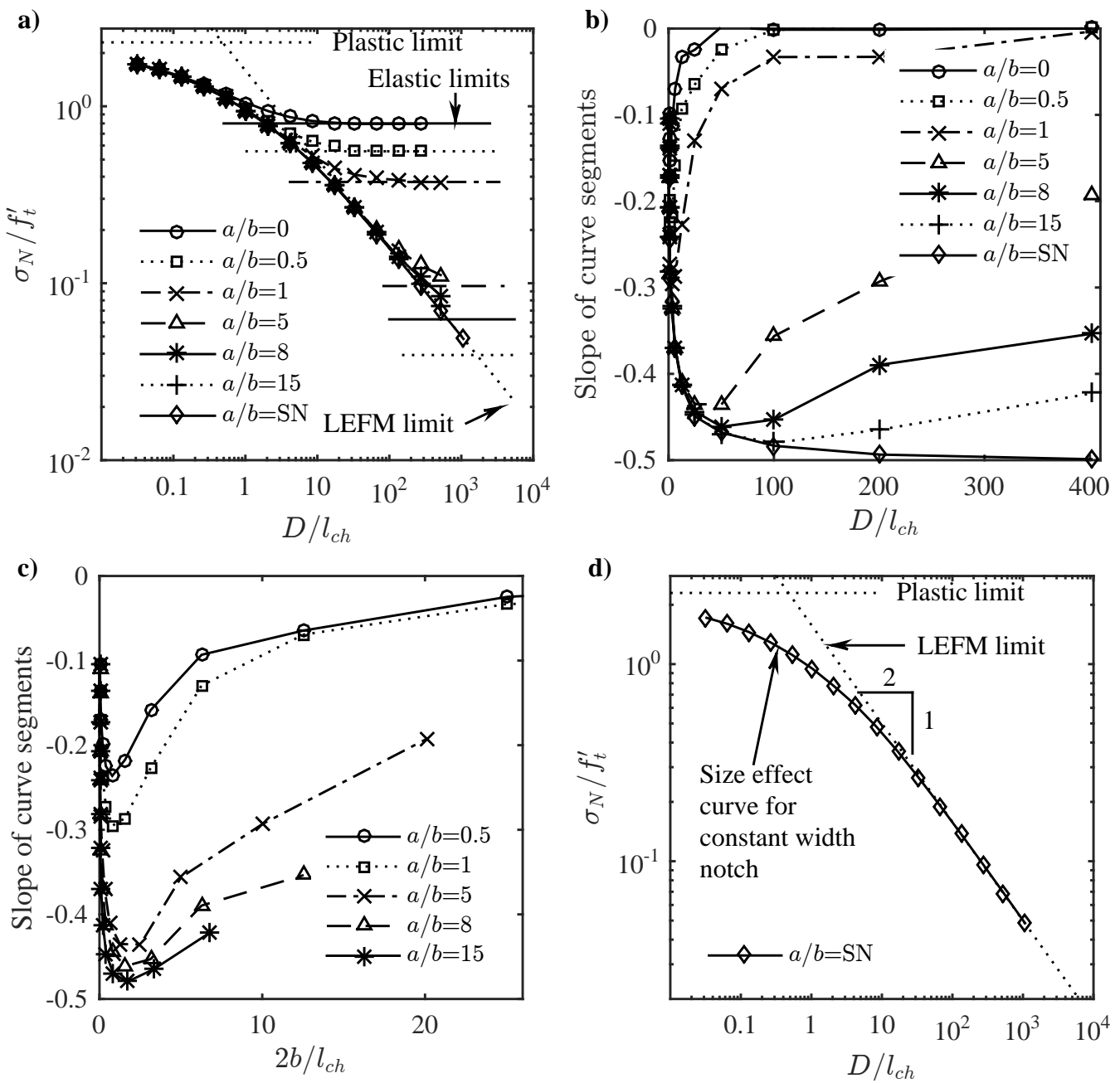

Figure 4: TPB Configuration: (a) size effect curves for elliptically notched geometries, (b) slope of size effect curves versus normalized size, (c) slope of size effect curves versus normalized width of elliptical notch, and (d) size effect curve for constant-width notch geometry. 
The plastic limit is defined as a stress state where the entire cross-section ahead of, and collinear with, the original notch is subject to a uniform stress state equal to the tensile strength, $f_{t}^{\prime}$.

For the CCP configuration, the nominal stress associated with the plastic limit can be calculated by considering the free-body diagram of one half of the panel with a uniform distribution of stress along the central line and by equating the forces acting at each end: $\sigma_{N} t D=f_{t}^{\prime}(1-\alpha) t D$. Substituting $\sigma_{N p}$ for $\sigma_{N}$ and rearranging leads to $\sigma_{N p}=f_{t}^{\prime}(1-\alpha)$, and so the normalized nominal stress associated with the plastic limit is $(1-\alpha)=0.875$.

The plastic limit behavior for the TPB configuration is less intuitive to calculate $[4,10]$. In the limit of $D \rightarrow 0$ the central cross section with the cohesive crack law consists of two parts: one of finite depth under tension with a perfectly plastic uniform distribution of stress and a concentrated force in the compressed zone which shrinks to a point. This is due to the fact that the smooth extrapolation to zero size must preserve the property that the compression stresses on the crack plane continue to be elastic during the entire loading process.

If one considers the TPB specimen shown in Fig. 2b, at the zero size limit, one has $\sigma=f_{t}^{\prime}=$ uniform distribution through the entire ligament except a concentrated compression force $F_{c}=t D(1-\alpha) f_{t}^{\prime}$ acting on the compression side. In this situation, the bending moment in the notched cross section is $M=0.5 t D^{2}(1-\alpha)^{2} f_{t}^{\prime}$, the applied load is $P_{\max }=4 M / S$, and the plastic limit results in $\sigma_{N u}=3(1-\alpha)^{2} f_{t}^{\prime}$. Consequently, the normalized nominal stress at the plastic limit is $3(1-\alpha)^{2}=2.2969$.

Finally, Fig. 3a and 4a report the LEFM size effect limit (Eq. 3) which 
plot as a straight line with a slope of $-1 / 2$ in the log-log scale.

In Fig. 3a, all of the calculated size effect curves for the CCP configuration tend to the plastic limit for $D \rightarrow 0$. As the sample size increases, they deviate from the plastic limit with slopes that become increasingly negative. In addition, each curve has an inflection point where the slope begins to become less negative and tends back towards zero. These changes in slope are portrayed in Fig. 3b where, for each curve segment of Fig. 3a, the average normalized size and slope are plotted as $[\mathrm{X}, \mathrm{Y}]$ pairs. The inflection points of the Fig. 3a curves correspond to the minimum slope values in the Fig. 3b plots.

Up to their individual inflection points, all curves in Fig. 3a are essentially coincident, and this "common" curve tends, for large enough $a / b$ values, to the LEFM limit. Past their inflection points, each curve departs from this common curve, and as $D \rightarrow \infty$, the size effect behavior tends to the corresponding elastic limit. Similarly to Fig. 3b, the slopes of the Fig. 3a curve segments were also plotted as a function of the normalized elliptical notch width, $2 b / l_{c h}$, and this is shown in Fig. 3c. In this figure, one can see that the minimum value of slope, which corresponds to the inflection point in the associated size effect curve, occurs at $2 b / l_{c h} \approx 2$. Based on these observations it is clear that the size effect behavior for arbitrary $a / b$ begins an asymptotic approach towards the LEFM limit, on a size effect path common to all $a / b$. However, as the elliptical notch width, $2 b$, reaches an approximate value of $2 l_{c h}$, the size effect behavior inflects away from the LEFM limit. As $a / b$ tends to infinity (i.e. a sharp-notched geometry), $b \approx 0$ for all $D$, and so the size effect behavior never deflects from the common curve. This is also 
consistent with the fact that for a sharp notch the stress is singular and the elastic limit is zero, making the horizontal asymptote in the log-log plot to be located at $\infty$.

This can be further verified by calculating the size effect curve for sharpnotch behavior obtained with a constant notch width of $4 \mathrm{~mm}\left(\ll 2.0 l_{c h} \approx\right.$ $300 \mathrm{~mm}$ ). The obtained size effect curve is presented in log-log scale in Fig. 3d. No deviation from an asymptotic approach towards the LEFM limit can be visually detected. Quantitatively, this is also the case, since the slope of the last three segments are $-0.491,-0.495$, and -0.498 .

Similarly to the CCP geometry also the TBP geometry displays the same behavior for blunt notches, i.e. for $a / b=$ constant. Fig. 4a shows all of the calculated size effect curves which tend to the plastic limit for $D \rightarrow 0$ and to the corresponding elastic limit for $D \rightarrow \infty$. Only the size effect curve for sharp-notched specimens approaches asymptotically towards the LEFM limit, see Figs. 4a and d.

Similarly, the size effect curves of the TPB configuration with blunt notch present an inflection point where the slope begins to become less negative and tends back towards zero. These changes in slope are portrayed in Figs. $4 \mathrm{~b}$ and $\mathrm{c}$ where the inflection points of the Fig. 3a curves correspond to the minimum slope values in the Fig. 3b plots. One can see in Fig. 3c that the minimum value of slope, which corresponds to the inflection point in the associated size effect curve, occurs again at $2 b / l_{c h} \approx 2$.

It is worth mentioning that similar studies on notch/defect sensitivity and on the transition from blunt to sharp notches for the fatigue behavior of metals have been carried out by various authors in the past [27, 28, 29, 30, 31]. 


\section{Fracture Process Zone Length}

An important indicator of the overall quality of the numerical simulations is the stress profiles at the crack centerline at peak load. Other than the slope discontinuity at the leading edge of the fracture process zone, where $\sigma_{x x}=f_{t}^{\prime}$, and in close proximity to the point load in a Three-Point Bend test, there should be no discontinuities in the stress field.

Any scatter, noise or fluctuation in the plots is an indication that the mesh size is too coarse for the interface elements along the crack path. As shown in Fig. 5 the stress profiles obtained in the numerical simulations are smooth and accurate.

The stress profiles along the crack path highlight also a key aspect of the behavior of quasi-brittle materials related to the FPZ, whose length does not scale with the size of the specimen.

The FPZ is the region on the stress plots where softening cohesive behavior occurs, i.e. from the notch tip $\left(y / D=\alpha_{0}=0.125\right.$, the dashed line in the plots of Fig. 5) to the point where $\sigma_{x x} / f_{t}^{\prime}=1$.

Let us consider first the behavior of TPB samples with sharp notches (Fig. 5a and b).

For a $D=5 \mathrm{~mm}$ curve, i.e. the smaller size considered in the numerical simulations, the FPZ comprises approximately $82 \%$ of the ligament depth, and as one can see in Fig. 5a, the stresses in the FPZ are not anywhere close to a fully-softened state. This is determined by noting where the stress profile intersects the horizontal line at $y / D=\alpha_{0}=0.125$. The value of the $x$-coordinate at this intersection gives the value of the principal normal stress at the crack tip as a percentage of the tensile strength, $f_{t}^{\prime}$. The stress at the 

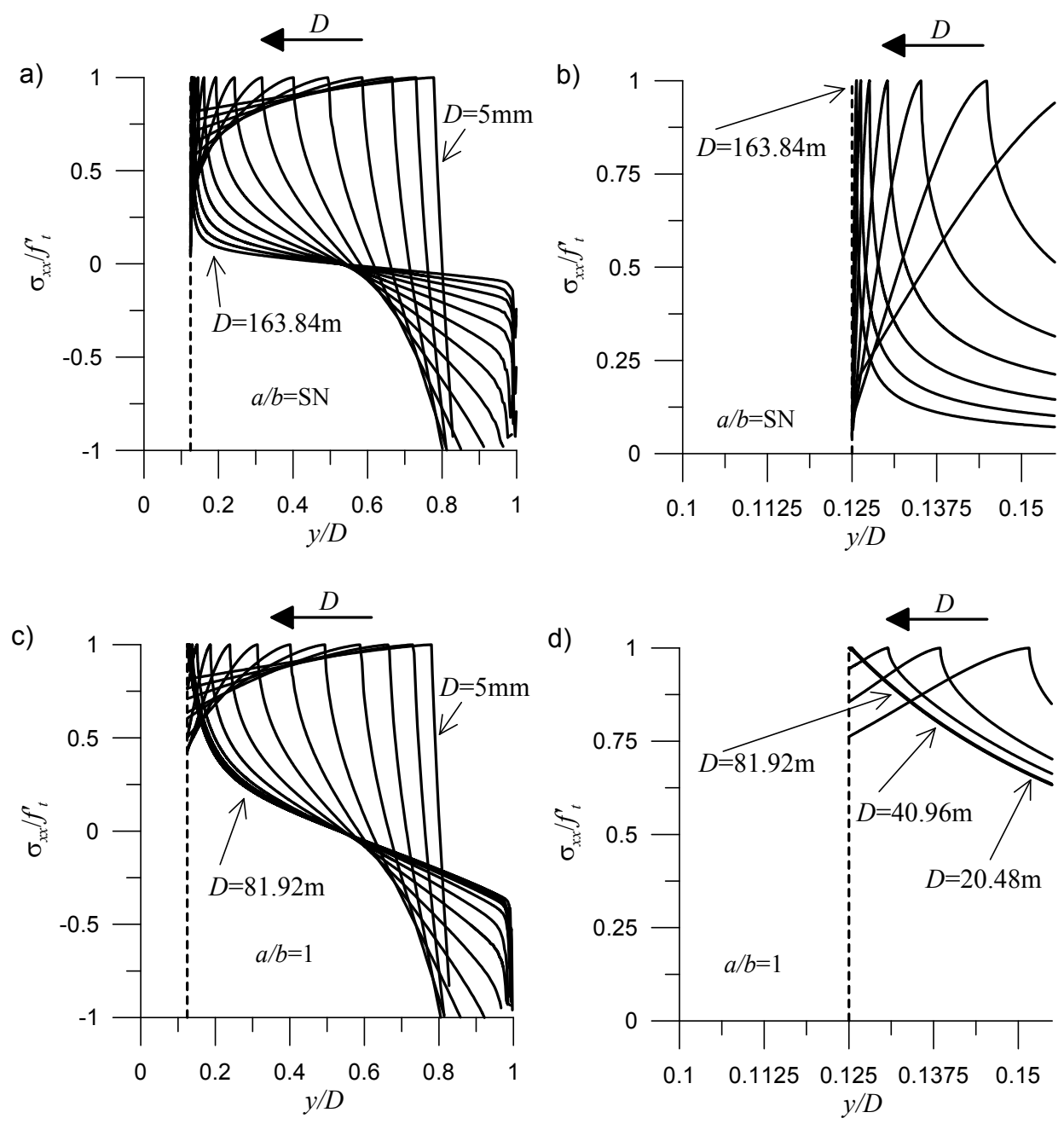

Figure 5: Stress profiles along the centerline at the peak load for TPB configuration with sharp notch (a) and its close-up at the tip (b), and with blunt notch of $a / b=1$, i.e. circular notch, (c) and its close-up at the tip (d). 

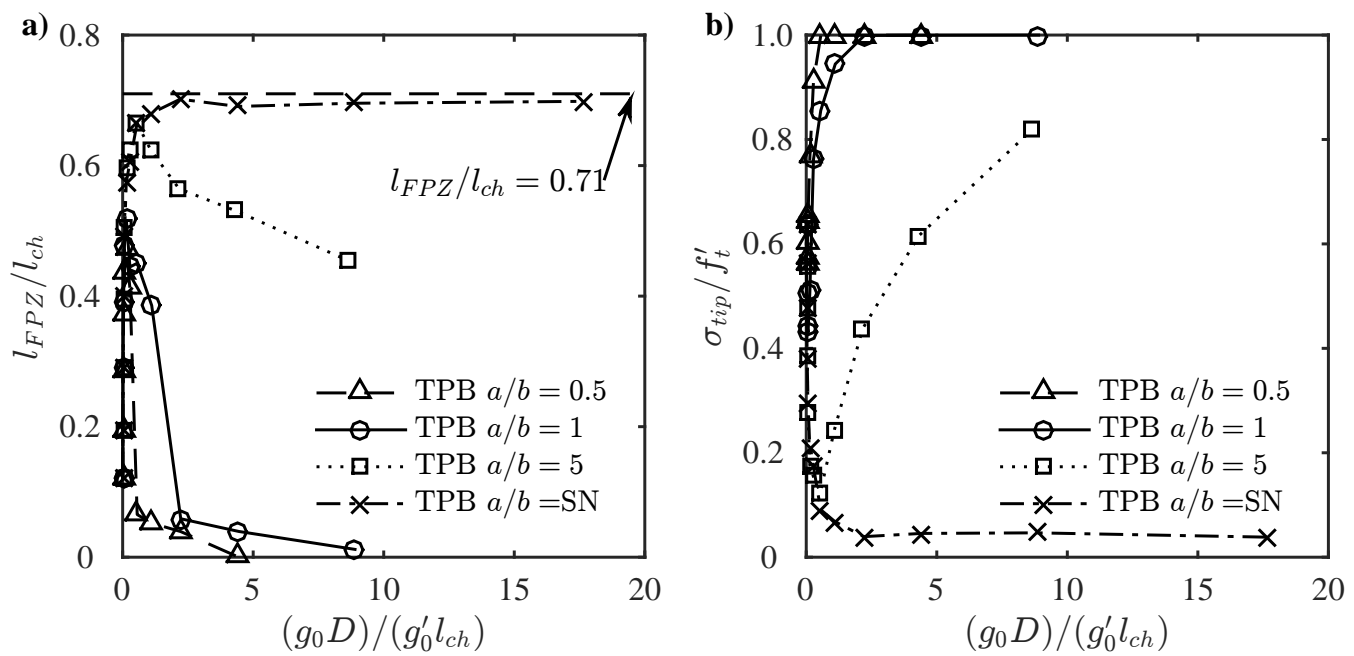

Figure 6: TPB configuration: normalized length of fracture process zone at peak load (a); normalized notch tip stress at peak load (b).

crack tip for $D=5 \mathrm{~mm}$ is over $80 \%$ of $f_{t}^{\prime}$.

For the largest sizes (see Fig. 5b for a close-up at the tip notch), the curve segments in and near the FPZ are indistinguishable since the size of the FPZ length at peak load tends to become constant for increasing size (Fig. 6a) and it occupies smaller and smaller percentage of the ligament depth. For $D=163,840 \mathrm{~mm}$, the FPZ extends to approximately $y=0.1257 D$, which corresponds to a FPZ length of $(0.1257-0.125) \times 163840 \approx 107 \mathrm{~mm}$ and only $0.0746 \%$ of the ligament depth.

For large size, the stress state in the FPZ symptotically approaches the fully-softened condition: for example, the stress at the notch tip for $D=$ $163,840 \mathrm{~mm}$ is $3.5 \%$ of $f_{t}^{\prime}$.

The normalized values (respect to the characteristic length) of the FPZ size at peak load are displayed in Fig. 6a. 
The evolution of the stress profile in the ligaments makes it evident why the nominal stress at peak load must decrease as the structure size increases. For the TPB configuration, the decrease in nominal stress manifests itself as a decreasing counter-clockwise rotation away from the $y$-axis for the bending stress profile as specimen size increases.

The specimens with blunt notch with different value of $a / b$ have a completely different asymptotic behavior for $D \rightarrow \infty$, as discussed in the Section 2. This is also confirmed by the the length of the FPZ and the stress at the notch tip at peak load. As shown in Figs. 6a and 5d the size of the FPZ at peak load shrinks and, for very large sizes, becomes almost a point. In addition, the stress at the notch tip tends to the tensile strength, $f_{t}^{\prime}$ (see Figs. $6 \mathrm{~b}$ and $5 \mathrm{~d}$. As a consequence, for specimens with blunt notch the size of the FPZ at peak load is no more a material property not even for large sizes in contrast to the case for sharp notches.

In Fig. 6a, the length of the fracture process zone (FPZ) at peak load is plotted for all data sets. Fig. 6b shows the stress at the notch tip for all data sets. Both figures display the concept that the length of the FPZ at peak load asymptotically approaches the fully-softened condition only for specimens with sharp notch. According to the literature, the size of the FPZ at peak load, for very large sizes, should be a material property [4]. Therefore, a quick review of Fig. 6 might suggest that the data is contradicting that principle: the curves for different values of $a / b$ appear to be approaching different asymptotes. However, the specimens with a sharp notch clearly have an asymptote of the size of the FPZ at peak load for $D \rightarrow \infty$ and the notch tip stress is closest to the fully-softened condition. Regardless of the 
details noted in the above paragraph, the length of the FPZ has, in general, leveled off by size $D=5,120 \mathrm{~mm}$, and the curve appear to be gradually approaching some limiting value (i.e. the fully-softened length) as $D \rightarrow \infty$. Since $c_{f}$ also approaches its limiting value as $D \rightarrow \infty$, it appears that the size range $D \geq 5,120 \mathrm{~mm}$ is appropriate for use in estimating $c_{f}$.

\section{GCSEC for a Linear Cohesive Crack Law}

An approximated analytical CSEC formula for TPB geometries with sharp notch was proposed in [1], inspired by the pioneering work of Planas [4], by interpolation of numerical results obtained with a linear cohesive law. That analytical expression was designed to match both the small-size (plastic limit) and large-size (asymptotic) behaviors and has the following expression

$$
\frac{f_{t}^{\prime 2}}{g_{0}^{\prime} \sigma_{N u}^{2}}=\frac{g_{0} D}{g_{0}^{\prime} l_{c h}}+\left(1+11 \sqrt{\frac{g_{0} D}{g_{0}^{\prime} l_{c h}}}\right)\left(\beta_{0}+25 \sqrt{\frac{g_{0} D}{g_{0}^{\prime} l_{c h}}}\right)^{-1}
$$

where $\beta_{0}=9(1-\alpha)^{4} g_{0}^{\prime}$ and the associated SEL is given in the Eq. 2 .

In this paper a generalization of the expression in Eq. 4 is proposed to include the size effect of structures with both sharp and blunt notches. Similarly to the previous work, the new analytical formula, entitled Generalized Cohesive Size Effect Curve (GCSEC) is obtained by the interpolation of numerical results obtained with the adoption of a linear cohesive law. Keeping in mind, as showed in the previous section, that for large size $(D \rightarrow \infty)$ the GCSEC formula must tends to the Bažant's SEL for sharp notches and to a horizontal asymptote, the elastic limit, for blunt notches. In addition, this analytical expression is formulated in a such way to match the plastic limit for small sizes. 
The GCSEC can be expressed by the following formula

$\sigma_{N u}=f_{t}^{\prime}\left(g_{0} \frac{D}{l_{c h}}+g_{0}^{\prime} \frac{1+11\left(\frac{g_{0} D}{g_{0}^{\prime} l_{c h}}\right)^{n}}{\beta_{p}^{2} g_{0}^{\prime}+25\left(\frac{g_{0} D}{g_{0}^{\prime} l_{c h}}\right)^{n}}\right)^{-1 / 2}\left(g_{0} \beta_{e}^{2} \frac{D}{l_{c h}}+\frac{1}{\left(1+g_{0} \beta_{e}^{2} \frac{D}{l_{c h}}\right)^{m}}\right)^{1 / 2}$

Alternatively, the GCSEC can be expressed similarly to CSEC in Eq. 4 as

$$
\frac{f_{t}^{\prime 2}}{g_{0}^{\prime} \sigma_{N u}^{2}}=\left(\frac{g_{0} D}{g_{0}^{\prime} l_{c h}}+\frac{1+11\left(\frac{g_{0} D}{g_{0}^{\prime} l_{c h}}\right)^{n}}{\beta_{p}^{2} g_{0}^{\prime}+25\left(\frac{g_{0} D}{g_{0}^{\prime} l_{c h}}\right)^{n}}\right)\left(g_{0} \beta_{e}^{2} \frac{D}{l_{c h}}+\frac{1}{\left(1+g_{0} \beta_{e}^{2} \frac{D}{l_{c h}}\right)^{m}}\right)^{-1}
$$

In both Eq. 5 and Eq. $6, \beta_{e} f_{t}^{\prime}$ and $\beta_{p} f_{t}^{\prime}$ are the elastic limit and the plastic limit nominal stresses, respectively; the parameters $n$ and $m$ depend on the geometry.

The GCSEC function is plotted in Fig. 7 for CCP geometry and in Fig. 8 for TPB geometry. The best fitting of the numerical data sets was obtained with $n=0.45 m=0.7$ and $n=2, m=0.9$ for CCP and TPB configuration, respectively. Fig. 7a for CCP geometry and in Fig. 8a for TPB geometry show clearly that the size effect curves merge in a single curve for different notch shape ratios $a / b$ and for $D / l_{c h}$ smaller than 1 . However, the approximation of the GCSEC formula is also good with regard to the slope of the numerical size effect curves as one can see from Figs. b and c in 7 for CCP geometry and in 8 for TPB geometry. In Fig. $7 d$ and in Fig. 8d the GCSEC and numerical data are plotted in the parametric space given by $X=g_{0} D /\left(g_{0}^{\prime} l_{c h}\right)$ and $Y=f_{t}^{\prime 2} /\left(g_{0}^{\prime} \sigma_{N}^{2}\right)$ showing again a good fit. In the small size range (Fig. 7 for CCP geometry and in Fig. 8 for TPB geometry), the behavior is shown to be different for the two structural configurations. However, the GCSEC formula is capable of capturing the 
two different asymptotic behavior and value, which differ from the Bažant's SEL (red straight line in the Figures).

Sharp notch - size effect of type II. In the case of sharp notch, i.e. $a / b \rightarrow \infty$, the LEFM gives an elastic limit $\sigma_{N e}=0$, and so $\beta_{e}=0$. This means that Eq. 5 as the following expression

$$
\sigma_{N u}=f_{t}^{\prime}\left(g_{0} \frac{D}{l_{c h}}+g_{0}^{\prime} \frac{1+11\left(\frac{g_{0} D}{g_{0}^{\prime} l_{c h}}\right)^{n}}{\beta_{p}^{2} g_{0}^{\prime}+25\left(\frac{g_{0} D}{g_{0}^{\prime} l_{c h}}\right)^{n}}\right)^{-1 / 2}
$$

For $D \rightarrow \infty$, the previous expression, Eq. 7 , gives

$$
\sigma_{N u}=f_{t}^{\prime}\left(g_{0} \frac{D}{l_{c h}}+\frac{11}{25} g_{0}^{\prime}\right)^{-1 / 2}
$$

This means that the GCSEC formula for sharp notches has a large-size (asymptotic) behavior coinciding with Bažant's SEL. At the same time, for $D \rightarrow 0$, the nominal strength, $\sigma_{N}$, in Eq. 7 , tends to the plastic limit, i.e. $f_{t}^{\prime} \beta_{p}$.

Blunt notch. In the case of blunt notch, for increasing size $D$ and arbitrary $a / b, \sigma_{N}$ begins an asymptotic approach towards the LEFM limit with a common path common to all $a / b$. However, as the elliptical notch width, $b$, reaches an approximate value of $l_{c h}$, the size effect behavior inflects away from the LEFM limit with a deviation of the GCSEC formula from the SEL to an horizontal asymptote which depends on the actual values of $a / b$ and $\alpha$. For $D \rightarrow \infty$ the the asymptotic value of Eq. 5 is

$$
\lim _{D \rightarrow \infty} \sigma_{N u}=f_{t}^{\prime}\left(g_{0} \frac{D}{l_{c h}}+\frac{11}{25} g_{0}^{\prime}\right)^{-1 / 2}\left(g_{0} \beta_{e}^{2} \frac{D}{l_{c h}}\right)^{1 / 2}=f_{t}^{\prime}\left(\beta_{e}^{2}\right)^{1 / 2}=f_{t}^{\prime} \beta_{e}
$$

It is worthwhile to remark that the normalized energy release rate, $g_{0}$, and its derivative, $g_{0}^{\prime}$, required for the blunt notch case are the LEFM reference 
values and they are calculated for the sharp notch case with the same $\alpha$, as for the GCSEC plotted in 7 for CCP geometry and in Fig. 8 for TPB geometry.

No notch - size effect of type I. The case of failure that occurs at crack initiation from a smooth surface is also considered in the GCSEC formula as a limit case when $a / b \rightarrow 0$. This case is taken into account in the Eq. 5 with the appropriate value for the elastic limit $\beta_{e}$. The asymptotic behavior for $D \rightarrow \infty$ of the GCSEC is the same of Eq. 9. For $D \rightarrow 0$, the nominal strength, $\sigma_{N}$, in Eq. 5, tends to the plastic limit, i.e. $f_{t}^{\prime} \beta_{p}$, like in the case of sharp notch. It is worth noting that both the size effect of type I and II due to the cohesive crack law tends, for $D \rightarrow 0$, to the same value, i.e. same plastic (or strength) limit. As it can be seen from Figs 7 and 8 for $\alpha=0.125$ the size effect behavior is always the same for $D / l_{c h}<1$. However, when $\alpha \rightarrow 0$ or $a / b \rightarrow 0$ the normalized energy release rate $g_{0}$ is equal to zero. As for the blunt notch, also in this case the normalized energy release rate, $g_{0}$, and its derivative, $g_{0}^{\prime}$, in Eq. 5 are obtained for the sharp notch case with a certain $\alpha$ and $a / b \rightarrow 0$. In any case the GCSEC in Eq. 5 gives the correct asymptotic behavior also for no notch structure. In fact for $g_{0} \rightarrow 0$ one gets

$$
\sigma_{N u}=f_{t}^{\prime}\left(g_{0} \frac{D}{l_{c h}}+\frac{1}{\beta_{p}^{2}}\right)^{-1 / 2}\left(g_{0} \beta_{e}^{2} \frac{D}{l_{c h}}+1\right)^{1 / 2}
$$

which for $D \rightarrow 0$ and for $D \rightarrow \infty$ gives $\sigma_{N}=f_{t}^{\prime} \beta_{p}^{2}$ and $\sigma_{N}=f_{t}^{\prime} \beta_{e}$, respectively, i.e. the plastic and elastic limit.

To highlight the accuracy of the proposed formula beyond the numerical simulations used for its development, the GCSEC was also utilized to fit the numerical results of Planas et al [3] on notched beams under TPB with 

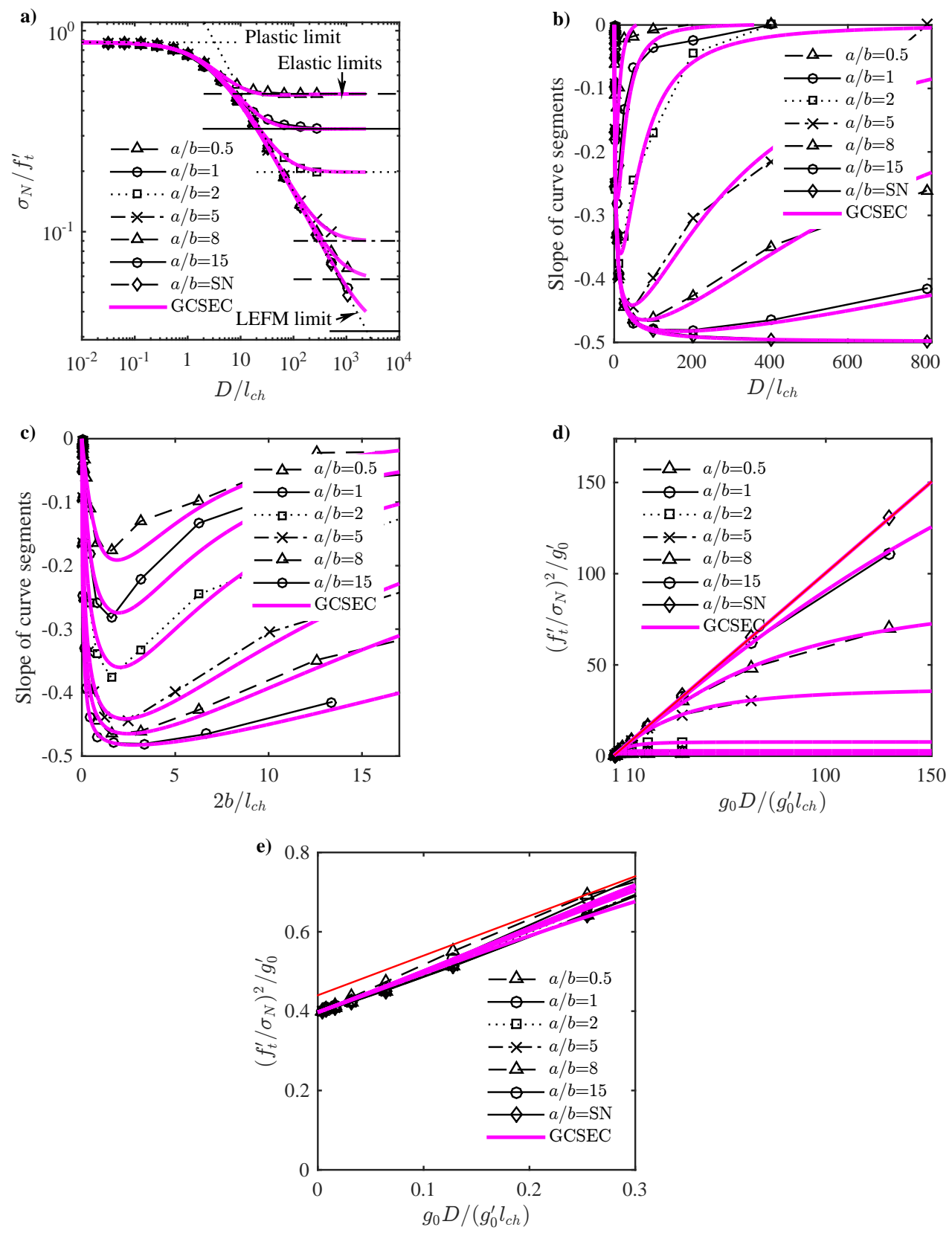

Figure 7: CCP Configuration: a) size effect curves for elliptically notched geometries; b) slope of size effect curves versus normalized size; c) slope of size effect curves versus normalized width of elliptical notch; d) size effect curve for constant-width notch geometry. 

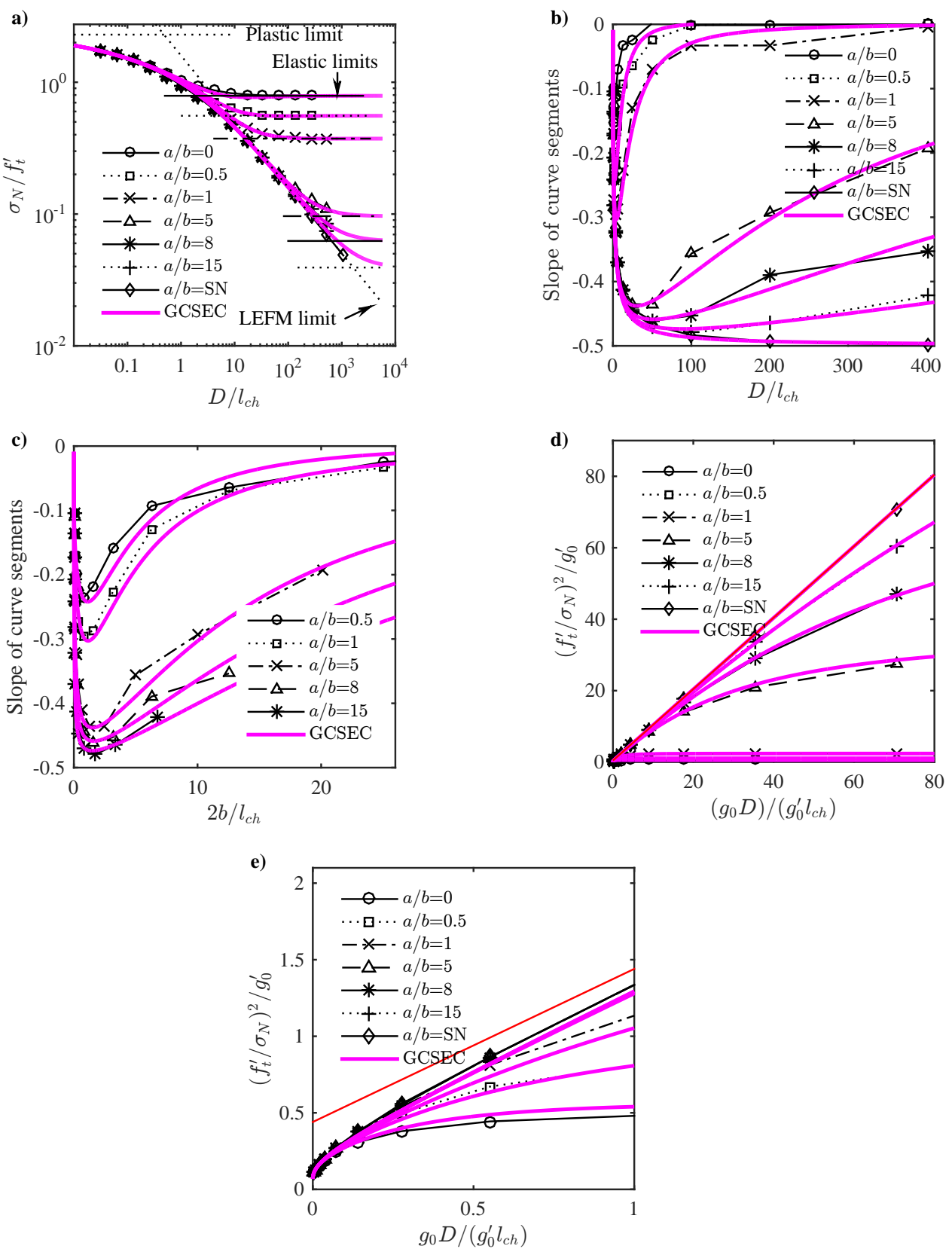

Figure 8: TPB Configuration: a) size effect curves for elliptically notched geometries; b) slope of size effect curves versus normalized size; c) slope of size effect curves versus normalized width of elliptical notch; d) size effect curve for constant-width notch geometry. 
linear softening. Fig. 9 shows the results for a beam with span-to-depth ratio of 4 and with different notch-to-depth ratio spanning from 0.1 to 0.7 . The proposed interpolating GCSEC equation fits accurately the numerical results for all notch depths assuming $n=0.45$ and $m=0.25$.

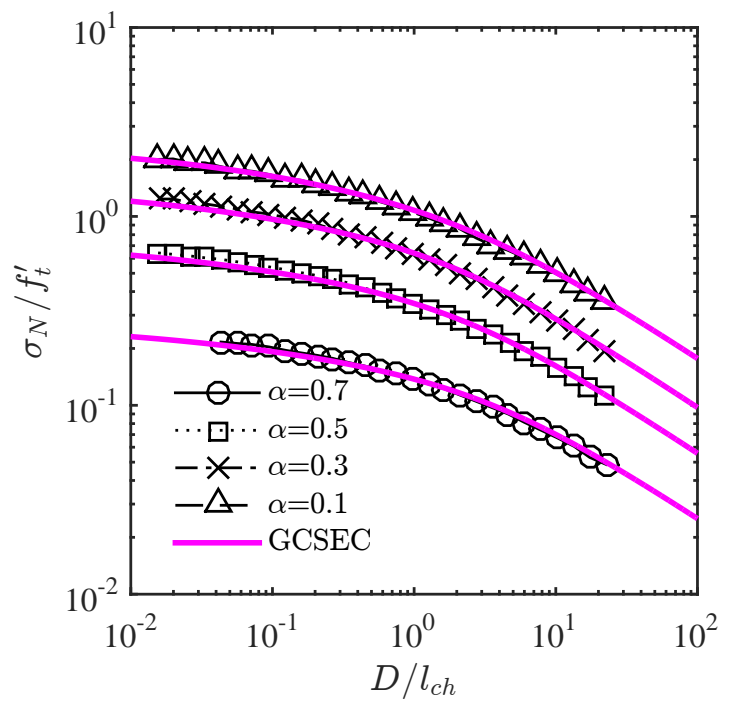

Figure 9: Comparison between the GCSEC formula and TPB numerical simulations for different notch-to-depth ratio of [3].

\subsection{Comparison with the Universal Size Effect Law}

Hoover and Bažant [33] formulated the so-called Universal Size-shape Effect Law (USEL) which describes the dependence of nominal strength on structural size and notch depth with the transition from Type 1 to Type 2 size effect. The deterministic version of the USEL reads

$$
\sigma_{N u}=\left[\frac{E G_{f}}{g_{0} D+(1-\lambda) c_{f} g_{0}^{\prime}+\lambda E G_{f} / f_{r \infty}^{2}}\right]^{-1 / 2}\left(1+\frac{r \lambda D_{b}}{\bar{D}+l_{p}}\right)^{1 / r}
$$


It is worth observing that the USEL was derived on the basis of asymptotic matching considerations and by fitting a large data set of experimental data. The derivation was completely independent on the cohesive crack model.

Figure 10 compares the USEL and the GCSEC for the two cases of TPB with no notch $\left(r=0.5, g_{0}=0, \lambda=1, \bar{D}=D, D_{b}=43 \mathrm{~mm}\right.$ and $\left.l_{p}=35 \mathrm{~mm}\right)$ and TPB with sharp notch $\left(r=0.5, \alpha=0.125, \lambda=0, g_{0}=0.353, g_{0}^{\prime}=2.732\right.$, $E=24,000 \mathrm{~N} / \mathrm{mm}^{2}, G_{f}=0.05 \mathrm{~N} / \mathrm{mm}, f_{t}^{\prime}=2.8 \mathrm{~N} / \mathrm{mm}^{2}$, and $c_{f}=0.2 l_{c h}$. . For large enough sizes the two curves are virtually indistinguishable. This demonstrates that, despite the completely different derivation, the USEL and the cohesive crack model provide equivalent results for large sizes. Shauffert and Cusatis [1] drew the same conclusion but limited to the case of sharp notches.

It is also instructive to observe in Fig. 10b the different behavior for very small size of the GCSEC and the USEL. In this case for $D \rightarrow 0$, contrarily to the GSEC, the USEL does not match the plastic limit.

\subsection{Comparison with Experimental Data}

Even though the objective of the current study was to study the size-effect predictions associated with the cohesive crack model rather them to validate the cohesive crack model against experimental data, it is still instructive to compare the accuracy of GSEC in fitting the nominal strengths of sample with different size and different notch depth.

Among the results available in the literature, the most exhaustive set of data is the one of Hoover et al [34] on concrete, which included tests on three-point-bending setups with various notch depths and four different sizes spanning a large size range of 1:12.5. Figure 11 shows the plot of experimental 

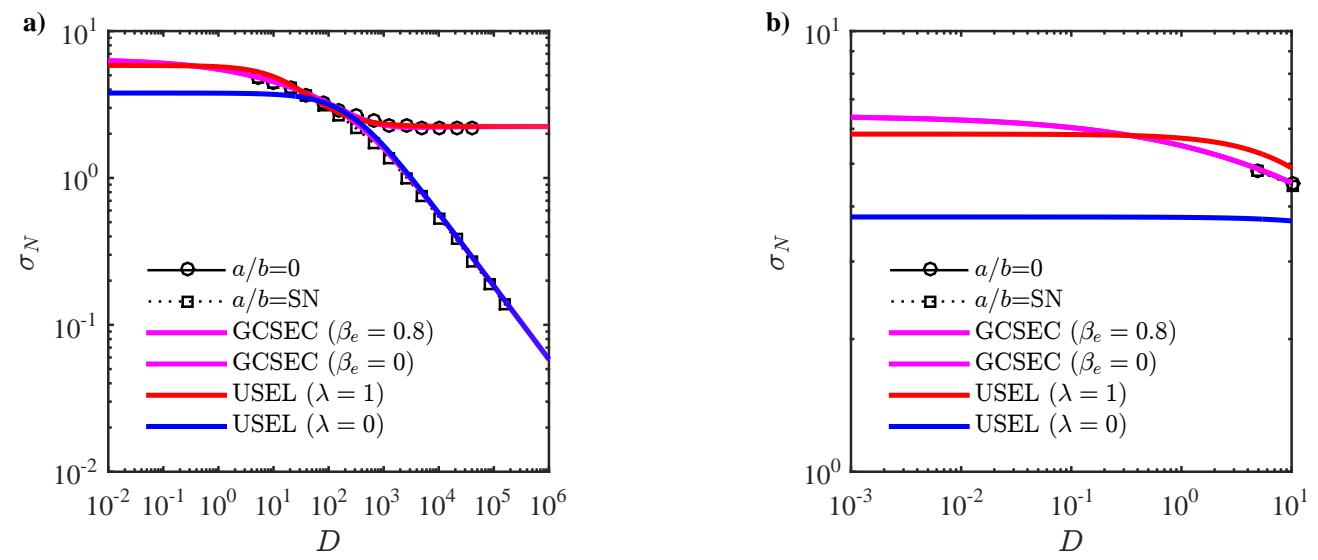

Figure 10: Comparison between the GCSEC and USEL for all the dimensional scale (a) and for very small scale (b).

nominal strength $\sigma_{N}$ versus the sample dimension $D$ and the the GCSEC best fitting. The best fitting was obtained with $f_{t}^{\prime}=4.5 \mathrm{MPa}, l_{c h}=119 \mathrm{~mm}$, $n=0.5$, and $m=0.42$ and as one can see the proposed interpolating GCSEC formula fits very well the experimental data for all notch depths greater than zero.

\section{Conclusions}

In this study, the size dependence of structural strength was analyzed via an extensive computational analysis of the size effect implied by a linear cohesive crack law. The primary objective of the study was to understand the effect of different types of notch: blunt and sharp. This work significantly extends the results of a previous study [1] for the following reasons. (a) The size effect behavior was studied for two different structural configurations 

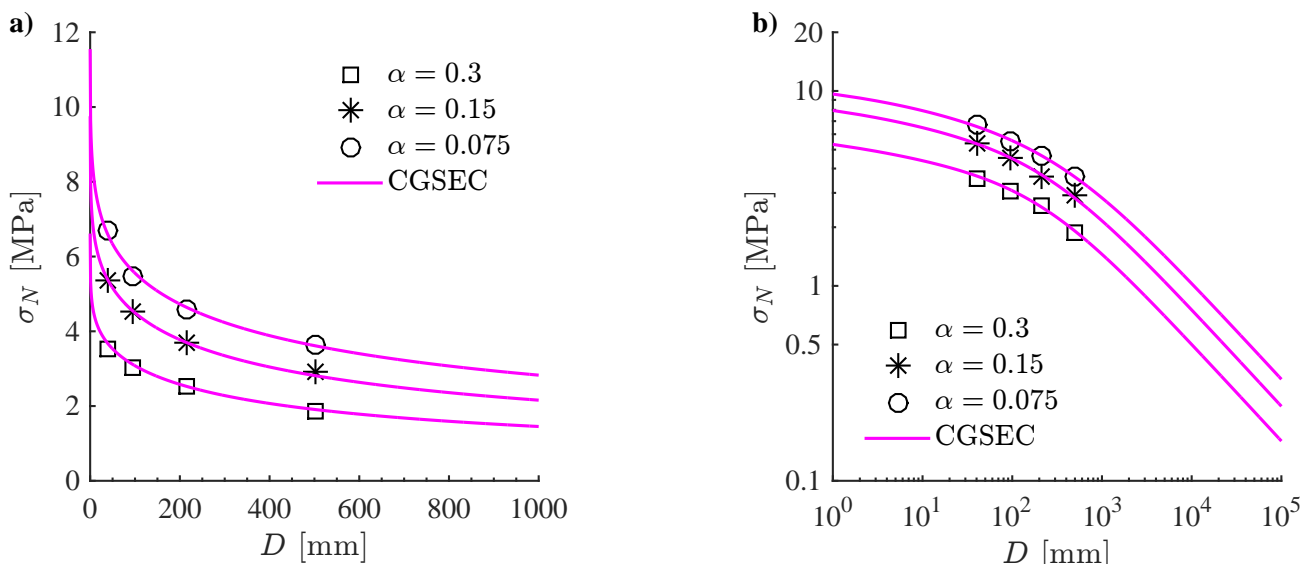

Figure 11: Comparison between the GCSEC formula and the TPB experimental data of [34] in natural (a) and logarithmic (b) scale.

(the three-point bending with span/depth ratio equal to 3 and the center crack panel) and featuring an elliptical blunt notch with constant aspect ratio to preserve geometric similarity. (b) The response associated with different values of the notch aspect ratio was calculated and compared with the size effect behavior of the same geometry with sharp notch.

Based on the obtained results, the following conclusions can be drawn

1. For specimens with blunt notches, the cohesive size effect curves are characterized by a continuous transition from the plastic behavior for small sizes to the purely brittle behavior with failure at crack initiation for large sizes. Hence, these size effect curves tend asymptotically to the elastic limit.

2. For specimens with blunt notches, in a double logarithmic plot, the cohesive size effect curves feature a point of inflection where the curve 
curvatures transition from negative to positive. At this inflection point the curve slope approaches the LEFM slope of $-1 / 2$ for increasing stress concentration at the notch tip.

3. The point of inflection occurs when the notch width is approximately equal to twice the material characteristic length, $2 l_{c h}$.

4. In the case of sharp notches, for which the elastic limit is zero, the elastic asymptote is located, theoretically, at $-\infty$ in a double logarithmic plot. In this condition the size effect curve does not have an inflection point and it approaches asymptotically the LEFM limit.

5. For sharp notches, the crack stress profiles at the peak load for different sizes reveal that the FPZ length converges to constant a value of $0.71 l_{c h}$ (for linear softening) and the and notch tip cohesive stress converges to zero as the structural size increases. For blunt notches, however, the behavior is completely different. In fact, at the peak load the length of the FPZ first increases and then it shrinks for increasing structural size. Asymptotically, it becomes a mathematical point and the stress at the notch tip tends to the tensile strength, $f_{t}^{\prime}$. As a consequence, for specimens with blunt notch and contrarily to the case of sharp notches, the size of the FPZ at peak load is not a material property.

6. The numerical results on structural strength can be approximated accurately with an analytical equation, entitled Generalized Cohesive Size Effect Curve (GCSEC), which is valid for different structural configurations with both blunt or sharp notch.

7. The GCSEC applies to both type I and II size effect.

8. The GCSEC depends on the cohesive crack parameters such as the 
tensile strength, $f_{t}^{\prime}$, the fracture energy, $G_{f}$, and, as a consequence, the characteristic length, $l_{c h}$. The GCSEC takes into account the effect of different structural configurations through the LEFM nondimensional energy release rate and its derivative.

9. The GSEC provides size effect estimates that agree very well with the Universal Size Effect Law and the associated experimental data.

\section{Acknowledgement}

. The work of the second author was supported partially by the U.S. National Science Foundation (NSF) under Grant No. CMMI-1435923. Preliminary work of Dr. Edward A. Schauffert on this project is acknowledged gratefully.

[1] Cusatis, G., Schauffert, E.A., 2009. Cohesive crack analysis of size effect. Engineering Fracture Mechanics, 76, 2163-2173.

[2] Hillerborg, A., Mooder, M., Petersson, P.E., 1976 Analysis of crack formation and crack growth in concrete by means of fracture mechanics and finite elements. Cement and Concrete Research, 6, 773-782.

[3] Planas J., Guinea G.V., Elices M., 1997.Generalized size effect equation for quasi-brittle materials. Fatigue \& Fracture of Engineering Materials \& Structures, 20(5), 671-687.

[4] Bažant, Z.P., Planas, J., 1998. Fracture and size effect in concrete and other quasibrittle materials. Boca Raton and London: CRC Press. 
[5] Cedolin, L., Cusatis, G. 2008. Identification of concrete fracture parameters through size effect experiments, Cement and Concrete Composites, 30(9), 788-797.

[6] Bažant Z.P., Kazemi M.T. 1990. Determination of fracture energy, process zone length and brittleness number from size effect, with application to rock and concrete. International Journal of Fracture 44(2), 111-131.

[7] Bažant Z.P., 1984. Size effect in blunt fracture: concrete, rock, metal. ASCE Journal of Engineering Mechanics, 110, 518-535.

[8] Planas J., Elices M., 1992. Asymptotic analysis of a cohesive crack 1. Theoretical background. International Journal of Fracture, 55, 153-177.

[9] Planas J., Elices M., 1993. Asymptotic analysis of a cohesive crack 2. Influence of the softening curve. International Journal of Fracture, 64, 221-237.

[10] Bažant, Z.P., Yu, Q., Zi, G., 2002. Choice of standard fracture test for concrete and its statistical evaluation. International Journal of Fracture, 118, 303-337.

[11] Schauffert, E.A., 2007. Fracture of quasi-brittle materials: size effect and the cohesive crack model. Master Thesis, Rensselaer Polytechnic Institute.

[12] Bažant, Z.P., 2002. Scaling of structural strength. Hermes-Penton Science: Springer. 
[13] Hughes, T.J.R., 2000. The finite element method: linear static and dynamic finite element analysis. Mineola, New York: Dover Publications.

[14] Bfer, G., 1985. An isoparametric joint/interface element for finite element analysis. International Journal for Numerical Methods in Engineering, 21(4),585-600.

[15] Gifford, L.N., Hilton, P.D., 1978. Stress intensity factors by enriched finite elements. Engineering fracture mechanics, 10, 485-496.

[16] Belytschko, T., Fish, J., Engelmann, B.E., 1988. A finite element with embeded localization zones. Computer methods in applied mechanics and engineering, 70, 59-89.

[17] Oliver, J., Cervera, M., Manzoli, O., 1999. Strong discontinuities and continuum plasticity models: the strong discontinuity approach. International journal of plasticity, 15, 319-351.

[18] Belytschko, T., Black, T., 1999. Elastic crack growth in finite elements with minimal remeshing. International journal for numerical methods in engineering, 45, 601620 .

[19] Moës, N., Dolbow, J., Belytschko, T., 1999. A finite element method for crack growth without remeshing. International journal for numerical methods in engineering, 46, 131-150.

[20] Moës, N., Belytschko, T., 2002. Extended finite element method for cohesive crack growth. Engineering fracture mechanics, 69, 813-833. 
[21] Tada, H., Paris, P.C., Irwin, G.R., 2000. The stress analysis of cracks handbook. New York: The American Society of Mechanical Engineers.

[22] Timoshenko, S. P., Goodier, J. N., 1970.Theory of elasticity. McGrawHill, New York.

[23] Planas, J., Guinea, G.V., Elices, M., 1995. Rupture modulus and fracture properties of concrete. In: Fracture Mechanics of Concrete Structures (Edited by F. H. Wittmann), Vol. I, pp. 95-110. Aedificatio Publishers, Freiburg, Germany.

[24] Young, W. C., Richard G. B., 2002. Roark's Formulas for Stress and Strain. Seventh Edition. McGraw-Hill.

[25] Inglis, C.E., 1913. Stresses in a plate due to the presence of cracks and sharp corners. Trans. Inst. Naval Archit., 55(1), 219-241.

[26] Neuber, H., 1958. Theory of Notch Stresses, Springer-VErlag, Berlin.

[27] Kitagawa H., Takahashi S., 1976. Applicability of fracture mechanics to very small cracks in the early stage, Proceedings of the 2nd International Conference on Mechanical Behaviour of Materials, 627-631.

[28] Smith R.A., Miller K.J., 1978. Prediction of fatigue regimes in notched components, International Journal of Mechanical Sciences, 20, 201-206.

[29] El-Haddad M.H., Topper T.H., Smith K.N., 1979. Prediction of NonPropagating Cracks, Engineering Fracture Mechanics, 11, 573-584. 
[30] Atzori B., Lazzarin P., 2001. Notch sensitivity and defect sensitivity under fatigue loading: two sides of the same medal International Journal of Fracture, 107(1), 1-8.

[31] Atzori B., Lazzarin P., Meneghetti G., 2003. Fracture mechanics and notch sensitivity. Fatigue \& Fracture of Engineering Materials \& Structures, 26, 257-267.

[32] Guinea, G. V., Pastor, J. Y., Planas, J., Elices, M., 1998. Stress intensity factor, compliance, and CMOD for a general three-point-bend beam. International Journal of Fracture, 89, 103-116.

[33] Hoover, C. G., Bažant, Z. P., 2014. Universal Size-Shape Effect Law Based on Comprehensive Concrete Fracture Tests. ASCE Journal of Engineering Mechanics, 140(3), 473-479.

[34] Hoover C. G., Bažant, Z. P., Vorel, J, Wendner, R, Hubler, M.H., 2013. Comprehensive concrete fracture tests: Description and results. Engineering Fracture Mechanics, 114, 92-103. 